\title{
THE
}

10-15-1993

\section{Electronic Band Structure of the Two-Dimensional Surface-State Bands of the $(1 \times 1)$ and (1×2) Phases of Bi/GaSb(110)}

D. N. Mcllroy

University of Rhode Island

David R. Heskett

University of Rhode Island, dheskett@uri.edu
A. B. McLean
R. Ludeke
H. Munekata

See next page for additional authors

Follow this and additional works at: https://digitalcommons.uri.edu/phys_facpubs

Terms of Use

All rights reserved under copyright.

\section{Citation/Publisher Attribution}

Mcllroy, D. N., Heskett, D., McLean, A. B., Ludeke,R., Munekata, H., \& DiNardo. N. J. (1993). Electronic band structure of the two-dimensional surface-state bands of the $(1 \times 1)$ and $(1 \times 2)$ phases of $\mathrm{Bi} / \mathrm{GaSb}(110)$. Physical Review B, 48(16), 11897-11904. doi: 10.1103/PhysRevB.48.11897

Available at: http://dx.doi.org/10.1103/PhysRevB.48.11897

This Article is brought to you for free and open access by the Physics at DigitalCommons@URI. It has been accepted for inclusion in Physics Faculty Publications by an authorized administrator of DigitalCommons@URI. For more information, please contact digitalcommons-group@uri.edu. 


\section{Authors}

D. N. Mcllroy, David R. Heskett, A. B. McLean, R. Ludeke, H. Munekata, and N. J. DiNardo 


\title{
Electronic band structure of the two-dimensional surface-state bands of the $(1 \times 1)$ and $(1 \times 2)$ phases of $\mathrm{Bi} / \mathrm{GaSb}(110)$
}

\author{
D. N. McIlroy* and D. Heskett \\ Department of Physics, University of Rhode Island, Kingston, Rhode Island 02881 \\ A. B. McLean \\ Department of Physics, Queen's University, Kingston, Ontario, Canada K7L $3 N 6$ \\ R. Ludeke and H. Munekata \\ IBM Thomas J. Watson Research Center, P. O. Box 218, Yorktown Heights, New York 10598 \\ N. J. DiNardo \\ Department of Physics and Atmospheric Science, Drexel University, Philadelphia, Pennsylvania 19104 \\ (Received 26 April 1993)

\begin{abstract}
The surface-state bands of the $(1 \times 1)$ and $(1 \times 2)$ phases of $\mathrm{Bi} / \mathrm{GaSb}(110)$ have been probed using angle-resolved ultraviolet photoemission spectroscopy with synchrotron radiation. Four Bi-induced surface-state bands have been identified for both the $(1 \times 1)$ and the $(1 \times 2)$ phases. The bands with the lowest binding energies ( $S^{\mathrm{I}}$ and $S^{\mathrm{II}}$ ) have been attributed to intrachain bonding in the $\mathrm{Bi}$ overlayer and the higher-binding-energy bands $\left(S^{\mathrm{III}}\right.$ and $\left.S^{\mathrm{IV}}\right)$ to overlayer states involved in the back bonding of the overlayer to the substrate. Based on initial-state dispersion measurements, we conclude that the $\mathrm{Bi}$ chains in the epitaxial overlayer remain intact throughout the phase transition. We propose a model for the overlayer structure of the $(1 \times 2)$ phase of $\mathrm{Bi} / \mathrm{GaSb}(110)$.
\end{abstract}

\section{INTRODUCTION}

Over the past decade the epitaxial overlayers formed by $\mathrm{Sb}$ and $\mathrm{Bi}$ on compound semiconductors have been a topic of intense interest. This is primarily due to the epitaxially ordered, atomically abrupt interfaces which form between both $\mathrm{Sb}$ and $\mathrm{Bi}$ on the (110) surfaces of many III-V compound semiconductors. ${ }^{1-5}$ Due to the covalent character of the semimetal-semiconductor bonds, information about overlayer-substrate bonding and structure can be understood by probing the surface-state bands.

The most extensively studied of these systems is $\mathrm{Sb} / \mathrm{GaAs}(110) .^{6-13}$ The accepted model of the overlayer structure of this system is the epitaxial continued layer structure (ECLS), which has been verified with lowenergy electron diffraction (LEED) and scanning tunneling microscopy (STM). ${ }^{1,2}$ In this model, the Sb overlayer consists of zigzag chains which run parallel to the [110] direction, and are registered above and between the GaAs substrate chains. However, recently there has been speculation regarding the universality of this structure in the case of $\mathrm{Bi}$ overlayers. ${ }^{9}$ In a tight-binding total-energy calculation by Bowler et al. ${ }^{14}$ of both the ECLS and epitaxial on-top structure (EOTS) of $\mathrm{Bi} / \mathrm{GaAs}(110)$, they have predicted an energy difference of only 0.06 $\mathrm{eV} /$ atom/unit cell between these two overlayer structures. This suggests that energetically favorable overlayer structures other than the ECLS may exist.

With LEED, ${ }^{15}$ dislocations have been observed every 12 adatoms along the overlayer chains of $\mathrm{Bi}$ on $\mathrm{GaAs}(110)$. The overlayer strain of $\mathrm{Bi} / \mathrm{GaAs}(110)$ is 0.16 , which is larger than the corresponding value of $\mathrm{Sb} / \mathrm{GaAs}(110)$ (0.11). Here we have quantified strain as the difference between the lattice spacing of the adatoms on the surface [which is equal to the substrate surface lattice spacing for a $(1 \times 1)$ overlayer] and their bulk lattice spacing divided by their bulk lattice spacing. It has been proposed that this larger mismatch between $\mathrm{Bi}$ and $\mathrm{GaAs}$ induces excessive strain within the overlayer which is relieved by the introduction of dislocations along the chains. ${ }^{16}$ Consequently, a compound semiconductor with a larger bulk lattice constant may better accommodate the larger size of $\mathrm{Bi}$ relative to $\mathrm{Sb}$, and thus eliminate the dislocations. This has been verified by STM of $\mathrm{Bi} / \mathrm{InAs}(110)$ where an epitaxially ordered overlayer, free of dislocations, has been observed. ${ }^{17}$ The overlayer strain of $\mathrm{Bi} / \operatorname{InAs}(110)$ is 0.10 , which is nearly identical to that of $\mathrm{Sb} / \mathrm{GaAs}(110)$.

These results suggest that overlayer strain due solely to a lattice mismatch may be insufficient to disrupt the epitaxial ordering of the semimetal overlayers provided the lattice mismatch is comparable to, or less than, that of $\mathrm{Sb} / \mathrm{GaAs}(110)$. Since the overlayer strain of $\mathrm{Bi} / \mathrm{GaSb}(110)$ is 0.10 , lattice-mismatch-induced strain may not be an issue when considering the overlayer transition from the $(1 \times 1)$ to the $(1 \times 2)$ phase. Meade and Vanderbilt ${ }^{18}$ have pointed out that a significant contribution to surface strain is the chemical interaction between the adsorbate and the substrate, which in turn will affect the hybridization of the overlayer-substrate bonds. The effects of overlayer strain on the structures of $\mathrm{Sb}$ and $\mathrm{Bi}$ overlayers on III-V(110) compound semiconductors has 
yet to be rigorously studied.

While $\mathrm{Sb}$ and $\mathrm{Bi}$ form $p(1 \times 1)$ overlayers on many of the (110) surfaces of III-V compound semiconductors at room temperature, $\mathrm{Bi} / \mathrm{GaSb}(110)$ is the only system which exhibits a surface phase transition from a $p(1 \times 1)$ to a $(1 \times 2)$ structure. Ford et al. ${ }^{15}$ observed that the commensurate $(1 \times 1)$ overlayer structure is metastable and can be driven into a stable incommensurate $(1 \times 2)$ structure by annealing at $200^{\circ} \mathrm{C}$. The doubling of the unit cell is perpendicular to the overlayer chains in the [001] direction [see Figs. 1(a) and 1(b)].

The fact that the overlayer makes the transition to the $(1 \times 2)$ phase by annealing at a temperature of only $200^{\circ} \mathrm{C}$ suggests that the metastable $(1 \times 1)$ structure may not be the ECLS and that an alternative overlayer structure may favor the more stable $(1 \times 2)$ phase. In addition, the overlayer-substrate bonding of $\mathrm{Bi} / \mathrm{GaSb}(110)$ may be significantly different from the other group-V semimetals on III-V(110) compound semiconductors. In the theoretical models of the overlayer structures of these systems, overlayer-induced surface-state bands have been attributed to either intrachain overlayer bonds which contribute to the bonding of the overlayer to the substrate, or intrachain bonds which do not. By probing the occupied surface-state bands of the $(1 \times 1)$ and $(1 \times 2)$ phases of $\mathrm{Bi} / \mathrm{GaSb}(110)$, we hope to identify which bonds are affected by the transition. This in turn should provide information about intrachain and overlayer-substrate bonding, as well as the structural changes which take place as a consequence of the transition from the $(1 \times 1)$ to the $(1 \times 2)$ phase.

\section{EXPERIMENTAL DETAILS}

The experiment was performed on beamline U12B at the National Synchrotron Light Source (NSLS), Brookhaven National Laboratory. The radiation was dispersed using a toroidal grating monochromator. ${ }^{19}$ The surfaces were prepared in a magnetically shielded UHV chamber with LEED and Ar-ion sputtering capabilities. The base pressure was $\leq 1 \times 10^{-10}$ Torr. The chamber was equipped with an angle-resolved hemispherical electrostatic energy analyzer ${ }^{20}$ with an angle of acceptance of $\pm 2^{\circ}$. The overall energy resolution was better than 200 meV. The sample was oriented such that the $\mathbf{A}$ vector of the incident photons was parallel to the mirror plane of the substrate [see Figs. 1(c) and 1(d)].

The sample consisted of an intrinsically doped, thick molecular-beam-epitaxial (MBE) -grown GaSb(110) film on an InAs(110) substrate. The sample was mounted on Ta foil which was supported by Ta wires. Current was passed through the Ta wires to resistively heat the sample. The temperature was monitored with a thermocouple attached to the Ta foil backing plate near the sample. The backing plate was mounted on a manipulator with rotational capabilities about two independent axes.

The sample was cleaned by $\mathrm{Ar}^{+}$sputtering followed by a $10-15 \mathrm{~min}$ anneal at $520^{\circ} \mathrm{C}$. This procedure resulted in reproducible valence spectra which compared favorably to previous photoemission spectra of Chiang and East$\operatorname{man}^{21}$ for a clean cleaved single crystal of $\mathrm{GaSb}(110)$, demonstrating the integrity of our MBE-grown sample and surface preparation technique.

Bi was evaporated onto the clean $\mathrm{GaSb}$ substrate from a boron-nitride effusion cell. During evaporation, the chamber pressure did not exceed $3 \times 10^{-10}$ Torr. After deposition, the sample was subsequently annealed at $100^{\circ} \mathrm{C}$ for $5 \mathrm{~min}$. The $p(1 \times 2)$-Bi phase was obtained by annealing the $p(1 \times 1)$-Bi overlayer at $250^{\circ} \mathrm{C}$ for $7 \mathrm{~min}$, at which time half-order spots appeared in the LEED pattern along the $\bar{\Gamma}-\bar{X}^{\prime}$ symmetry line. The Bi thickness was determined from timed exposures to the $\mathrm{Bi}$ evaporant beam. Deposition rates were established with a crystalthickness monitor. We have assumed a linear relation-

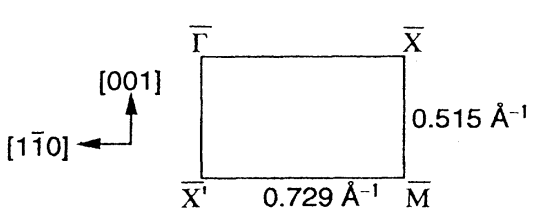

(a)

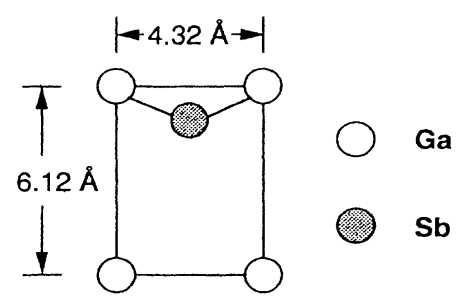

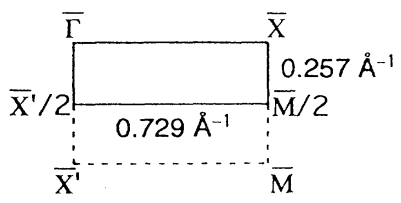

(b)

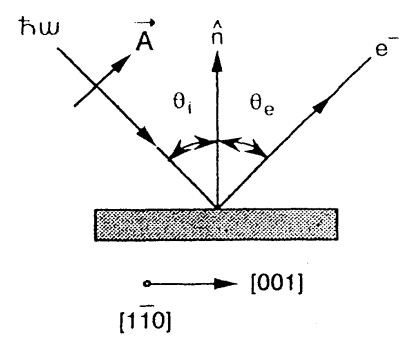

(d)
FIG. 1. (a) The surface Brillouin zone of $\mathrm{GaSb}(110)$. (b) The reduced surface Brillouin zone of the $p(1 \times 2)$ phase of $\mathrm{Bi} / \mathrm{GaSb}(110)$. (c) The real-space unit cell of $\mathrm{GaSb}(110)$. (d) $\mathrm{A}$ diagram of the experimental geometry. 
ship between the sticking coefficient of Bi to the sample and the thickness monitor. We estimate the accuracy of the overlayer to within $25 \%$, with a reproducibility of approximately $10 \%$. One monolayer of $\mathrm{Bi}$ is defined as two adatoms per unit cell of the $\mathrm{GaSb}(110)$ substrate $\left(7.57 \times 10^{14} / \mathrm{cm}^{2}\right)$. The integrated intensity of the $\mathrm{Bi} 5 d$ core level was observed to decrease by $5-15 \%$ during the overlayer transition from the $(1 \times 1)$ to the $(1 \times 2)$ phase. This is well within the experimental uncertainty of determining the completion of the first monolayer. Therefore, we have attributed the decrease in the $\mathrm{Bi} 5 d$ core-level intensity associated with the overlayer transition from the $(1 \times 1)$ to the $(1 \times 2)$ phase with the desorption of $\mathrm{Bi}$ exceeding one monolayer.

All the photoemission spectra presented in this paper and used to carry out our analysis of this system were recorded at a photon energy of $36 \mathrm{eV}$. Due to interference from photoemission peaks excited with second-order light from the monochromator, we found only a very narrow window around $36 \mathrm{eV}$ which yielded useful spectra for the valence band.

\section{RESULTS}

\section{A. The $p(1 \times 1)$ phase of $\mathrm{Bi} / \mathrm{GaSb}(110)$}

We have probed the occupied two-dimensional surface bands of the $(1 \times 1)$ and $(1 \times 2)$ phases of $\mathrm{Bi} / \mathrm{GaSb}(110)$ using angle-resolved photoemission. The experimental geometry is presented in Fig. 1(d). The photoemission spectra have been acquired along the high-symmetry lines of the surface reciprocal lattice [see Figs. 1(a) and 1(b)]. In this manner it is possible to map the two-dimensional band dispersion of the Bi-induced surface states. The parallel component of the wave vectors of the photoelectrons are calculated using the standard equation, $k_{\|}=(2 m E / \hbar)^{1 / 2} \sin \theta_{e}$, where $k_{\|}$is the component of the photoelectron's wave vector parallel to the surface, $E$ is the kinetic energy of the electrons, $m$ is the electron's mass, $\hbar$ is Planck's constant divided by $2 \pi$, and $\theta_{e}$ is the angle of emission of the photoelectrons with respect to the sample normal.

In Fig. 2 we present the normalized photoemission energy distribution curves of the valence-band spectra of clean $\mathrm{GaSb}(110)$ and the $p(1 \times 1)$ phase of $\mathrm{Bi} / \mathrm{GaSb}(110)$. The spectra were acquired at the $\bar{X}^{\prime}$ point of the surface Brillouin zone (SBZ) using a photon energy of $36 \mathrm{eV}$ at an incident angle of $65^{\circ}$, and are referenced to the experimentally determined Fermi level of the sample holder. Similar to $\mathrm{Bi} / \mathrm{GaAs}(110)$ (Ref. 16) and $\mathrm{Bi} / \mathrm{InAs}(110),{ }^{22}$ it is at the $\bar{X}^{\prime}$ zone boundary of the SBZ that the Bi-induced surface states are observable simultaneously. Unlike previously studied systems where only three Bi-induced states are observed, in the case of $\mathrm{Bi} / \mathrm{GaSb}(110)$ four $\mathrm{Bi}$ induced states have been identified. Following an earlier convention, ${ }^{6,8}$ we have labeled these states from lower to higher binding energy as $S^{\mathrm{I}}(-0.60 \mathrm{eV}), S^{\mathrm{II}}(-1.22 \mathrm{eV})$, $S^{\mathrm{III}}(-1.83 \mathrm{eV})$, and $S^{\mathrm{IV}}(-2.76 \mathrm{eV})$.

In Fig. 2 we have labeled the single substrate feature $A$. We attribute this feature at least partially to emission from a $\mathrm{GaSb}(110)$ surface state associated with the $\mathrm{Sb}$

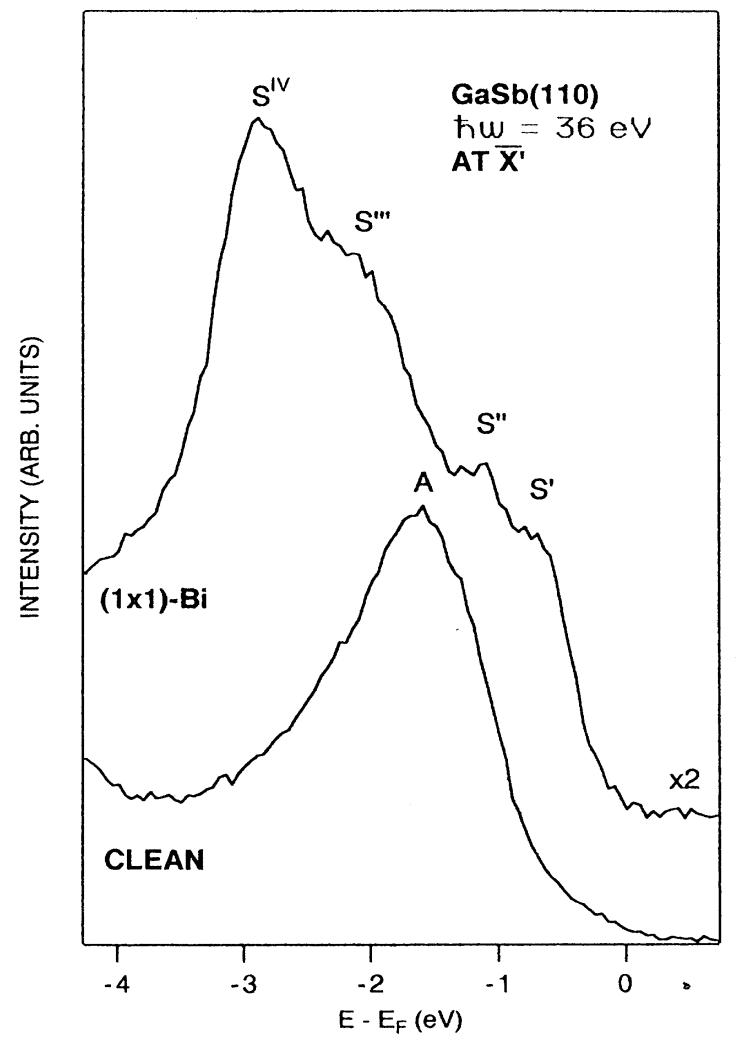

FIG. 2. Angle-resolved photoemission spectra of clean $\mathrm{GaSb}(110)$ and of monolayer coverages of the $(1 \times 1)$ phase of $\mathrm{Bi} / \mathrm{GaSb}(110)$ at the zone boundary $\bar{X}^{\prime}$.

dangling bond, in analogy with our previous studies of $\mathrm{GaAs}(110)$ (Ref. 16) and $\operatorname{InAs}(110),{ }^{22}$ and in agreement with experimental ${ }^{23,24}$ and theoretical ${ }^{8}$ investigations of $\mathrm{GaSb}(110)$. This peak is at least partially quenched upon the deposition of $\mathrm{Bi}$, which supports this assignment. In the case of $\mathrm{Bi} / \operatorname{InAs}(110),{ }^{22}$ the corresponding substrate feature was at the same binding energy as a probable Biinduced surface state. Consequently, it was not possible to verify that this state was specific to the Bi overlayer. This is not the case with $\mathrm{Bi} / \mathrm{GaSb}(110)$, where none of the Bi-induced surface states are at the same binding energy as the substrate feature $A$. It is for this reason that we can identify four Bi-induced surface states rather than three.

Similar to $\mathrm{Bi} / \mathrm{GaAs}(110)$ (Ref. 16) and $\mathrm{Bi} / \mathrm{InAs}(110),{ }^{22}$ the overlayer-induced surface states of $\mathrm{Bi} / \mathrm{GaSb}(110)$ are better resolved in the second SBZ along the $\bar{X}^{\prime}-\bar{\Gamma}_{2}$ direction. In Fig. 3 we present a sequence of valence-band spectra of the $(1 \times 1)$ phase of $\mathrm{Bi} / \mathrm{GaSb}(110)$ acquired along the $\bar{X}^{\prime}-\bar{\Gamma}_{2}$ azimuth. A comparison of Fig. 3 with a similar set of spectra of $\mathrm{Bi} / \mathrm{InAs}(110)$ (Ref. 22) demonstrates the similarities between these two systems. In Fig. 3 we see that $S^{\mathrm{I}}$ and $S^{\mathrm{II}}$ disperse very little across the $\bar{X}^{\prime}$ $\bar{\Gamma}_{2}$ region of the SBZ. In contrast, $S^{\mathrm{III}}$ and $S^{\mathrm{IV}}$ have significantly larger dispersions. 


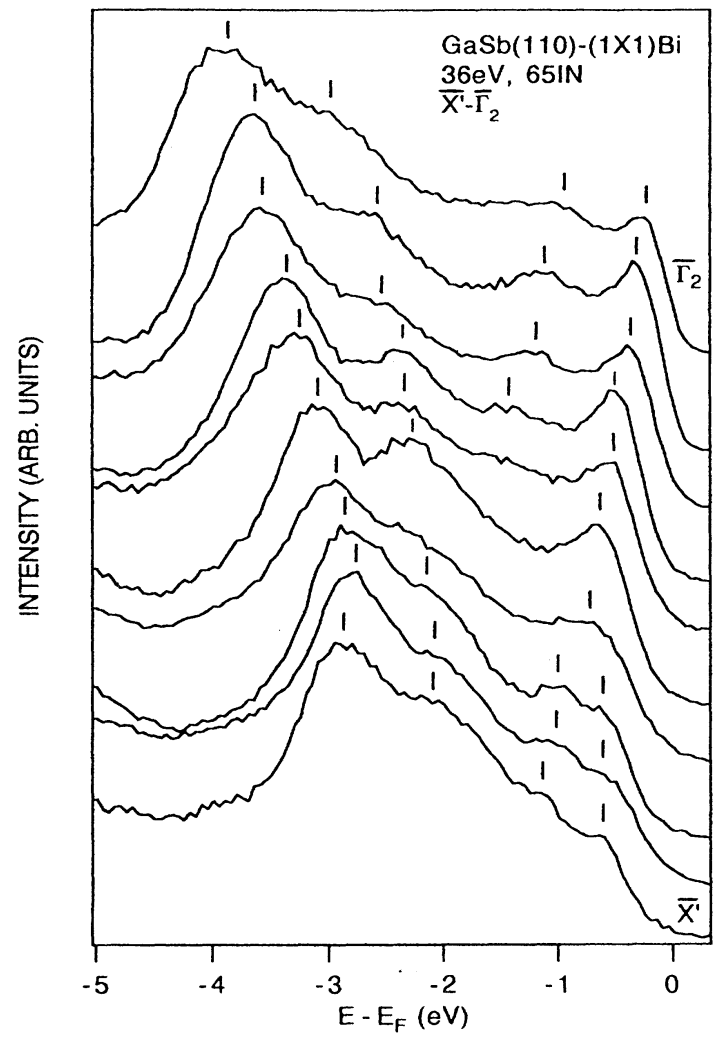

FIG. 3. Angle-resolved photoemission spectra of the valence band of monolayer coverages of the $(1 \times 1)$ phase of $\mathrm{Bi} / \mathrm{GaSb}(110)$ acquired along the $\bar{X}^{\prime}-\Gamma^{\prime}{ }_{2}$ azimuth of the second surface Brillouin zone.

In Fig. 4 we present the complete initial-state dispersion of the surface bands of the $(1 \times 1)$ phase of $\mathrm{Bi} / \mathrm{GaSb}(110)$. The symbols represent the $\mathrm{Bi}$-induced surface bands, while the hatched areas are the theoretical bulk band projections. ${ }^{8}$ Unlike $\mathrm{Bi} / \mathrm{GaAs}(110)$ (Ref. 16) and $\mathrm{Bi} / \mathrm{InAs}(110),{ }^{22}$ where there is substantial overlap between the bulk bands of the substrate and the higherbinding-energy $\mathrm{Bi}$-induced surface states, which makes mapping difficult, the surface bands of $\mathrm{Bi} / \mathrm{GaSb}(110)$ can be resolved across the entire SBZ. The positions of the surface states were determined by fitting the valence-band spectra with Gaussian distributions on top of linear backgrounds.

$\mathrm{Bi} / \mathrm{GaSb}(110)$ is the only system of group-V elements on III-V(110) compound semiconductors reported so far where $S^{\mathrm{I}}$ and $S^{\mathrm{II}}$ are resolved along the $\bar{\Gamma}-\bar{X}$ azimuth. It is not until the $\bar{X}$ zone edge that $S^{\mathrm{I}}$ and $S^{\mathrm{II}}$ become indistinguishable. Similar to $\mathrm{Bi} / \mathrm{InAs}(110),{ }^{22} S^{\mathrm{I}}$ and $S^{\mathrm{II}}$ appear as one degenerate, or nearly degenerate, band along the $\bar{X}-\bar{M}-\bar{X}^{\prime}$ directions. While $S^{\mathrm{I}}$ and $S^{\mathrm{II}}$ are relatively dispersionless along the $\bar{\Gamma}-\bar{X}^{\prime}$ azimuth, from Fig. 3 we see that they disperse significantly in the $\bar{\Gamma}-\bar{X}$ direction. The opposite is true of $S^{\mathrm{III}}$ and $S^{\mathrm{IV}}$. These states disperse significantly in the $\bar{\Gamma}-\bar{X}^{\prime}$ direction, but disperse much less in the $\bar{\Gamma}-\bar{X}$ direction. In Table I we have summarized the bandwidths of the surface bands of the $(1 \times 1)$ phase of

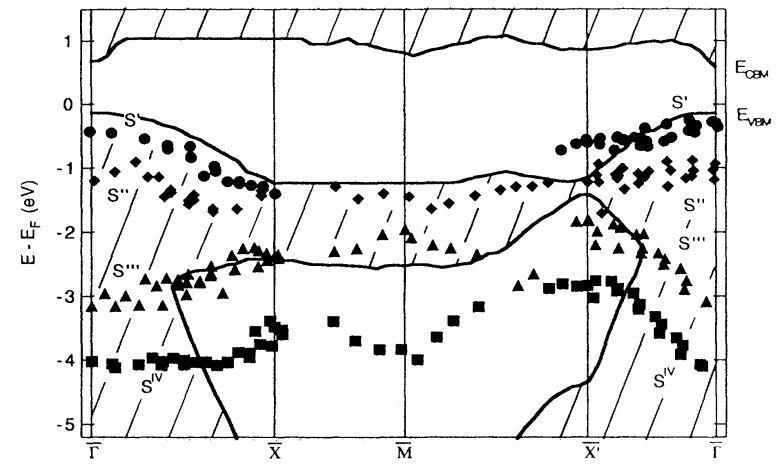

FIG. 4. The dispersion of the surface-state bands of monolayer coverages of the $(1 \times 1)$ phase of $\mathrm{Bi} / \mathrm{GaSb}(110)$ referenced to the sample Fermi level. The symbols are the experimentally determined dispersions of $S^{\mathrm{I}}, S^{\mathrm{II}}, S^{\mathrm{III}}$, and $S^{\mathrm{IV}}$. The hatched regions are the theoretical bulk band projections from Ref. 8 of clean $\mathrm{GaSb}(110)$.

$\mathrm{Bi} / \mathrm{GaSb}(110)$ across the $\bar{\Gamma}-\bar{X}$ and the $\bar{\Gamma}-\bar{X}^{\prime}$ azimuths. The significance of the bandwidths of the surface bands will be addressed in the discussion.

\section{B. The $p(1 \times 2)$ phase of $\mathrm{Bi} / \mathrm{GaSb}(110)$}

From analysis of the photoemission intensities of the Bi $5 d, \mathrm{Ga} 3 d$, and $\mathrm{Sb} 4 d$ core levels, we have determined that the $(1 \times 2)$ overlayer phase corresponds to a coverage of one monolayer of $\mathrm{Bi}$. This is consistent with the findings of a LEED study of $\mathrm{Bi} / \mathrm{GaSb}(110){ }^{15}$ In Fig. 5 we present spectra of the $(1 \times 1)$ and $(1 \times 2)$ phases of $\mathrm{Bi} / \mathrm{GaSb}(110)$ acquired near the second zone edge $\bar{\Gamma}_{2}$ of the $(1 \times 1)$ SBZ. We have borrowed the convention applied to the surface states of the $(1 \times 1)$ phase and labeled the states of the $(1 \times 2)$ phase from lower to higher binding energy as $S^{\mathrm{I}}, S^{\mathrm{II}}, S^{\mathrm{III}}$, and $S^{\mathrm{IV}}$. When we compare the spectra of the $(1 \times 2)$ phase with that of the $(1 \times 1)$ phase, we see that $S^{\mathrm{I}}$ and $S^{\mathrm{II}}$ persist after the surface structure transition, with $S^{\text {II }}$ shifting by $\sim 200 \mathrm{meV}$ to higher binding energy. Two higher-binding-energy states have been observed for the $(1 \times 2)$ phase, but are not easily resolved in the second zone of the SBZ, and subsequently are not observed in the spectrum of the $(1 \times 2)$ phase presented in Fig. 5.

In Fig. 6 we present the dispersion of the twodimensional surface bands of the $(1 \times 2)$ phase of $\mathrm{Bi} / \mathrm{GaSb}$ (110) across the reduced SBZ [see Fig. 1(b)]. The symbols are the experimentally determined $\mathrm{Bi}$-induced

TABLE I. The bandwidths of the surface-state bands of the $(1 \times 1)$ and $(1 \times 2)$ phases of $\mathrm{Bi} / \mathrm{GaSb}(110)$.

\begin{tabular}{lcccc}
\hline \hline & $(1 \times 1)$ & $\bar{\Gamma}-\bar{X}$ & $\begin{array}{c}\bar{\Gamma}-\bar{X}^{\prime} \\
(1 \times 1)\end{array}$ & $\begin{array}{c}\bar{\Gamma}-\bar{X}^{\prime} / 2 \\
(1 \times 2)\end{array}$ \\
\hline$S^{\text {I }}$ & $1.09 \mathrm{eV}$ & $0.83 \mathrm{eV}$ & $0.31 \mathrm{eV}$ & $0.10 \mathrm{eV}$ \\
$S^{\mathrm{II}}$ & $0.35 \mathrm{eV}$ & $0.79 \mathrm{eV}$ & $0.19 \mathrm{eV}$ & $0.04 \mathrm{eV}$ \\
$S^{\mathrm{III}}$ & $0.68 \mathrm{eV}$ & $0.19 \mathrm{eV}$ & $1.26 \mathrm{eV}$ & $0.16 \mathrm{eV}$ \\
$S^{\text {IV }}$ & $0.59 \mathrm{eV}$ & & $1.31 \mathrm{eV}$ & $0.12 \mathrm{eV}$ \\
\hline \hline
\end{tabular}




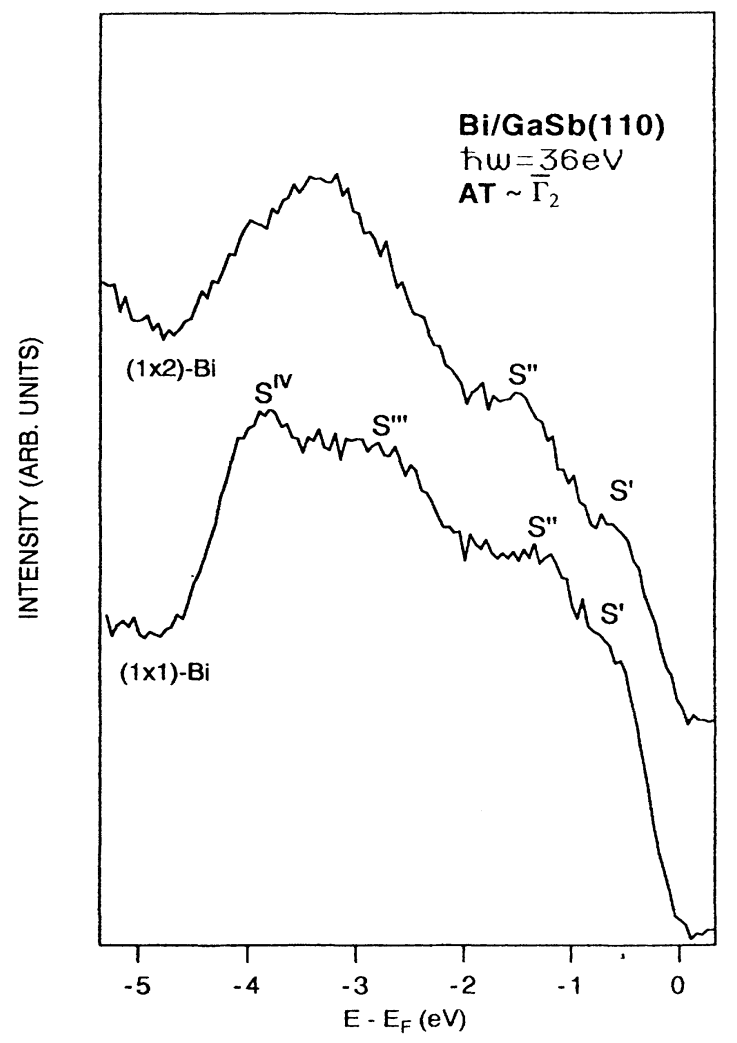

FIG. 5. Angle-resolved photoemission spectra of monolayer coverages of the $(1 \times 1)$ and $(1 \times 2)$ phases of $\mathrm{Bi} / \mathrm{GaSb}(110)$ near the second zone edge $\bar{\Gamma}_{2}\left(k_{\|}=1.24 \AA^{-1}\right)$.

surface bands and the hatched areas are the theoretical bulk band projections of $\mathrm{GaSb}(110){ }^{8}$ The bulk band projections along the $\bar{M} / 2-\bar{X}^{\prime} / 2$ symmetry line are not available; consequently, we only show the experimentally determined surface-band dispersions for this zone in Fig. 6. Similar to the $(1 \times 1)$ phase, the dispersion of the lower-binding-energy surface bands of the $(1 \times 2)$ phase can almost be mapped across the entire SBZ. Since the binding energy of $S^{\mathrm{I}}, S^{\mathrm{II}}, S^{\mathrm{III}}$, and $S^{\mathrm{IV}}$ of the $(1 \times 1)$ and $(1 \times 2)$ phases are almost equivalent at $\bar{\Gamma}$ of the SBZ, it is possible to make a one-to-one correspondence between these bands of the two phases.

The most pronounced difference between the band structure of the $(1 \times 2)$ and the $(1 \times 1)$ phases is the breaking of the degeneracy between $S^{\mathrm{I}}$ and $S^{\mathrm{II}}$ at the zone boundary, $\bar{X}$, after the overlayer phase transition. This suggests that a larger asymmetry exists between the two types of bonding of the overlayer to the substrate, i.e., $\mathrm{Bi}-\mathrm{Ga}$ versus $\mathrm{Bi}-\mathrm{Sb}$, or that there are changes in the bonding of the overlayer chains to the substrate. Across the $\bar{\Gamma}-\bar{X}^{\prime} / 2$ azimuth, the bandwidths of $S^{\mathrm{I}}$ and $S^{\mathrm{II}}$ decrease in the $(1 \times 2)$ phase relative to their corresponding widths in the $(1 \times 1)$ phase. In Table I we have summarized the bandwidths of $S^{\mathrm{I}}$ and $S^{\mathrm{II}}$ in both the $(1 \times 1)$ and $(1 \times 2)$ phases. With the exception of the bandwidth of $S^{\mathrm{II}}$ across the $\bar{\Gamma}-\bar{X}$ direction, which increases from 0.35 to $0.79 \mathrm{eV}$, the bandwidths of $S^{\mathrm{I}}$ and $S^{\mathrm{II}}$ are observed to de-

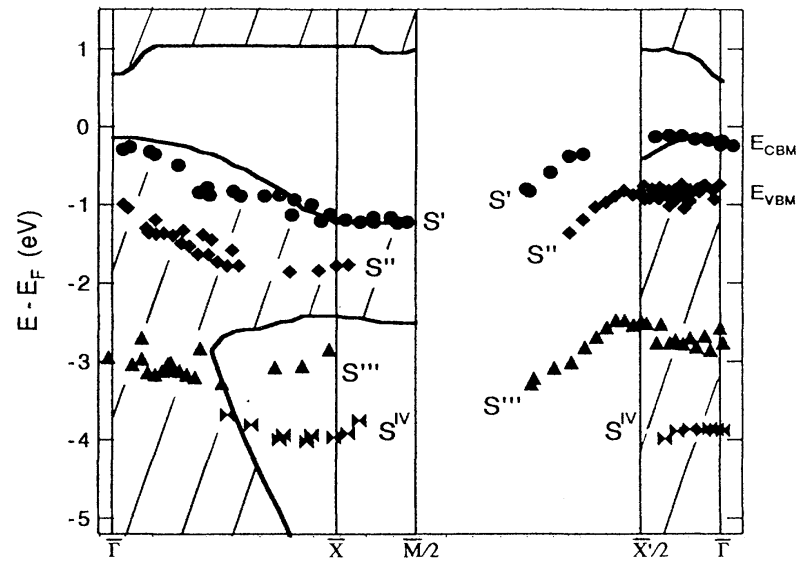

FIG. 6. The dispersion of the surface-state bands of monolayer coverages of the $(1 \times 2)$ phase of $\mathrm{Bi} / \mathrm{GaSb}(110)$ referenced to the sample Fermi level. The symbols are the experimentally determined dispersions of $S^{\mathrm{I}}, S^{\mathrm{II}}, S^{\mathrm{III}}$, and $S^{\mathrm{IV}}$. The hatched regions are the theoretical bulk band projections from Ref. 8 of clean $\mathrm{GaSb}(110)$.

crease as the overlayer passes through the phase transition from the $(1 \times 1)$ to the $(1 \times 2)$ structure.

Two bands $S^{\mathrm{III}}$ and $S^{\mathrm{IV}}$ are not as well resolved in the $(1 \times 2)$ phase as they were in the $(1 \times 1)$ phase. The overlayer transition appears to have had a much stronger affect on these two bands than on $S^{\mathrm{I}}$ and $S^{\mathrm{II}}$. The bandwidths of $S^{\text {III }}$ and $S^{\text {IV }}$ decrease significantly as the overlayer passes through the transition to the $(1 \times 2)$ phase (see Table I). In fact, the dispersion of $S^{\mathrm{III}}$ and $S^{\mathrm{IV}}$ by $\sim 0.60 \mathrm{eV}$ to halfway across the $\bar{\Gamma}-\bar{X}^{\prime}$ zone of the $(1 \times 1)$ phase is substantially larger than their bandwidths of 0.16 and $0.12 \mathrm{eV}$, respectively, along the $\bar{\Gamma}-\bar{X}^{\prime} / 2$ direction of the $(1 \times 2)$ phase. These results, as well as those presented above, should provide useful insight into the effects of the transition on the overlayer intrachain and overlayersubstrate bonds, as well as the nature of these bonds.

\section{DISCUSSION}

\section{A. The surface bands of the $p(1 \times 1)$ phase of $\mathrm{Bi} / \mathrm{GaSb}(110)$}

There are presently two schools of thought regarding the assignment of the surface bands of group-V semimetals on III-V(110) compound semiconductors. In a number of models (ECLS and EOTS), the two uppermost surface bands, which we have designated as $S^{\mathrm{I}}$ and $S^{\mathrm{II}}$, are overlayer states which contribute to overlayer-substrate bonding, and the higher-binding-energy bands $S^{\text {III }}$ and $S^{\text {IV }}$ are overlayer intrachain bonds which do not contribute to overlayer-substrate bonding. ${ }^{7-9,14}$ Others have arrived at the opposite conclusion. ${ }^{6,25}$ Consequently, the assignment of which overlayer bands contribute to the bonding of the $\mathrm{Bi}$ chains to the substrate, and which do not, has yet to be resolved. We have inferred that the $\mathbf{B i}$ adatoms couple to form chain structures on $\mathrm{GaSb}(110)$ in the $(1 \times 1)$ phase based on strong similarities between the dispersion of the surface bands of this system and those 
of $\mathrm{Sb} / \mathrm{GaAs}(110),{ }^{12} \mathrm{Bi} / \mathrm{GaAs}(110),{ }^{16}$ and $\mathrm{Bi} / \mathrm{InAs}(110),{ }^{22}$ where the formation of chains has been verified with STM. $^{2,3,17}$

It has been argued that dispersion along the $\bar{\Gamma}-\bar{X}^{\prime}$ direction is due to substrate-mediated interaction between the chains. ${ }^{14}$ However, the large bandwidths of $S^{\text {III }}(1.26 \mathrm{eV})$ and $S^{\mathrm{IV}}(1.31 \mathrm{eV})$ of the $(1 \times 1)$ phase of $\mathrm{Bi} / \mathrm{GaSb}(110)$ along the $\bar{\Gamma}-\bar{X}^{\prime}$ symmetry line, which is in the direction perpendicular to the $\mathrm{Bi}$ chains in real space, suggests that there must be considerable interaction between the overlayer chains and the substrate. It is unclear if this is indeed the case for $\mathrm{Bi} / \mathrm{GaSb}(110)$, or for $\mathrm{Bi} / \mathrm{InAs}$ (110) (Ref. 22) and $\mathrm{Bi} / \mathrm{GaAs}$ (110) (Ref. 16) which exhibit the same behavior, since this suggests that the substrate-mediated interactions are more extensive than intrachain interactions. It can be argued that the zigzag nature of the Bi chains will result in some dispersion perpendicular to the chains. This may be the case with $S^{\mathrm{IV}}$, which from Fig. 4 we see disperses almost equivalently along the $\bar{M}-\bar{X}^{\prime}$ direction as along the $\bar{\Gamma}-\bar{X}^{\prime}$ direction. Even if there is some dispersion perpendicular to the chains, as a consequence of the zigzag geometry, we expect the electrons within this band to be more delocalized in the direction parallel to the chains than perpendicular to them. If we also consider the fact that the bandwidths of these states are almost a factor of 2 smaller in the $\bar{\Gamma}-\bar{X}$ direction (see Table I), which is in the direction of the $\mathrm{Bi}$ chains in real space, we are inclined to conclude that these bands are involved in the bonding of the overlayer to the substrate.

Upon examination of the bandwidths of $S^{\mathrm{I}}$ and $S^{\mathrm{II}}$ in Table I, we see that these bands disperse more in the $\bar{\Gamma}-\bar{X}$ azimuth $(1.09$ and $0.35 \mathrm{eV})$ than in the $\bar{\Gamma}-\bar{X}^{\prime}$ azimuth $(0.31$ and $0.19 \mathrm{eV})$. This behavior has also been observed for the corresponding states of $\mathrm{Bi} / \operatorname{InAs}(110) .^{22}$ The larger dispersions of $S^{\mathrm{I}}$ and $S^{\mathrm{II}}$ in the direction of the chains suggests that these bands are localized along the $\mathrm{Bi}$ chains and are not heavily involved in overlayersubstrate bonding.

\section{B. The surface bands \\ of the $p(1 \times 2)$ phase of $\mathrm{Bi} / \mathrm{GaSb}(110)$}

The system of $\mathrm{Bi} / \mathrm{GaSb}(110)$ is a very useful system to study for two reasons: (1) Of all the systems of group-V semimetals on III-V(110) compound semiconductors, $\mathrm{Bi} / \mathrm{GaSb}(110)$ is the only one which passes through a surface phase transition from a metastable $(1 \times 1)$ structure to a stable $(1 \times 2)$ structure $^{15}(2)$ The $(1 \times 2)$ phase corresponds to a monolayer coverage of $\mathrm{Bi}$. If we first assume that the Bi overlayer consists of chains, then the doubling of the surface unit cell in the [001] direction, while the

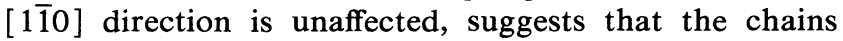
remain intact. This is supported by polarization studies of $\mathrm{Bi} / \mathrm{GaSb}(110)$ where only small changes are observed between the polarization dependence of the surface state bands of the $(1 \times 1)$ and $(1 \times 2)$ phases. $^{26}$

Bowler et al. ${ }^{14}$ find from a total-energy minimization calculation that the EOTS and ECLS of $\mathrm{Bi} / \mathrm{GaAs}(110)$ differ in energy per adatom per unit cell by only $0.06 \mathrm{eV}$. The annealing temperature of $250^{\circ} \mathrm{C}$ needed to drive the
$(1 \times 1)$ phase of $\mathrm{Bi} / \mathrm{GaSb}(110)$ to the $(1 \times 2)$ phase gives a rough estimate of $0.045-\mathrm{eV} /$ adatom/unit cell for the energy difference between these two phases. While we are not suggesting that the overlayer geometries of either of the two phases is necessarily the ECLS or EOTS, this comparison does give us an idea of the energies involved.

The transition from the $(1 \times 1)$ to the $(1 \times 2)$ phase may involve the coupling of neighboring chains. A doubling up of the Bi chains would probably have only a small effect on the bands associated with intrachain bonding. Larger effects may be expected for overlayer bands which contribute to overlayer-substrate bonding which may become strained in order to allow the Bi chains to couple. An alternative scheme for generating the $(1 \times 2)$ overlayer structure could involve the buckling of every other chain, but this is unlikely since there is no reasonable explanation for asymmetric buckling of every other chain. In order to maintain the unit cell in the [110] direction after the phase transition, it would be necessary for the buckling to be uniform along the chains.

In Fig. 6 we see that the two uppermost surface bands $S^{I}$ and $S^{\text {II }}$ remain intact. From Table I we observe that the dispersions of $S^{\mathrm{I}}$ and $S^{\mathrm{II}}$ actually increase. This supports our earlier conjecture that these bands should be attributed to intrachain bonds which do not contribute to overlayer-substrate bonding. At the same time, we see that $S^{\mathrm{I}}$ and $S^{\mathrm{II}}$ are no longer degenerate at the $\bar{X}$ point of the SBZ, relative to the $(1 \times 1)$ phase. In theoretical band-structure calculations of isolated $\mathrm{Sb}$ chains, Mailhiot, Duke, and $\mathrm{Chadi}^{8}$ found that the lower-bindingenergy states were degenerate at the band edge $\bar{X}$ of the SBZ. Only until the Sb chains were brought into contact with the substrate was this degeneracy lifted as a consequence of the asymmetry of the bonding of the chains to the different constituents of the substrate. We propose that the breaking of degeneracy between $S^{\mathrm{I}}$ and $S^{\mathrm{II}}$ of the $(1 \times 2)$ phase of $\mathrm{Bi} / \mathrm{GaSb}(110)$ is analogous to the above results by Mailhiot, Duke, and Chadi. ${ }^{8}$ We therefore suggest that the $(1 \times 2)$ structure increases the asymmetry within the $\mathrm{Bi}$ chains due either to changes in the interaction of the $\mathrm{Bi}$ adatoms with the $\mathrm{GaSb}(110)$ substrate, or to chain-chain interactions.

We can still identify what appear to be the original surface-state bands $S^{\mathrm{III}}$ and $S^{\mathrm{IV}}$ of the $(1 \times 1)$ phase in the $(1 \times 2)$ phase. Upon comparing their dispersion in Fig. 6 with their corresponding dispersion in the $(1 \times 1)$ phase (see Fig. 4), we see that they are not as well resolved. In addition, the dispersion of $S^{\mathrm{III}}$ and $S^{\mathrm{IV}}$ halfway along the $\bar{\Gamma}-\bar{X}^{\prime}$ azimuth is $\sim 0.60 \mathrm{eV}$, which is substantially larger than their bandwidths of 0.16 and 0.12 $\mathrm{eV}$, respectively, across the reduced $\bar{\Gamma}-\bar{X}^{\prime} / 2$ zone of the $(1 \times 2)$ phase. If $S^{\mathrm{III}}$ and $S^{\mathrm{IV}}$ of the $(1 \times 1)$ phase are attributable to intrachain bonds with very little involvement in overlayer-substrate bonding, we would expect them to be relatively unaffected by the transition, similar to what we have observed for $S^{\mathrm{I}}$ and $S^{\mathrm{II}}$. Since $S^{\mathrm{III}}$ and $S^{\text {IV }}$ do change significantly, this supports our earlier suggestion that these two bands of the $(1 \times 1)$ phase are involved in the bonding of the overlayer to the substrate, which we do expect to be strongly affected by the overlayer transition. 


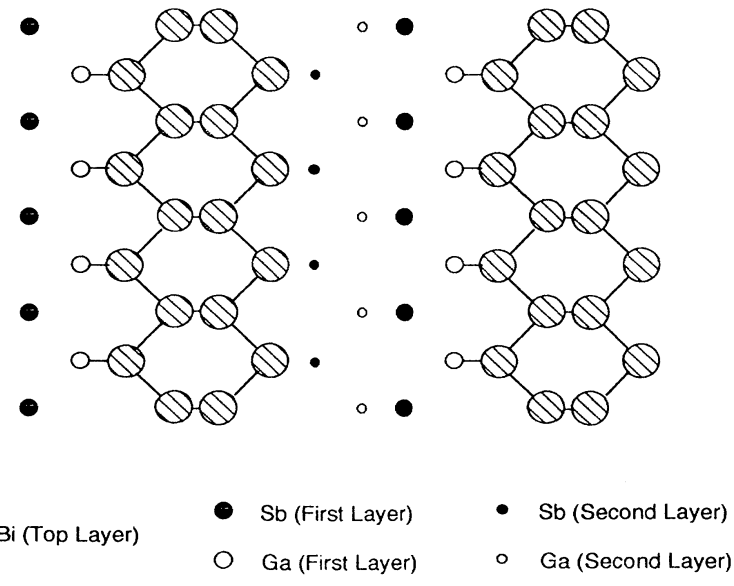

FIG. 7. A proposed geometry of the $(1 \times 2)$ phase of $\mathrm{Bi} / \mathrm{GaSb}(110)$ utilizing a combination of the ECLS and EOTS models to couple neighboring chains.

In light of the surface band-structure measurements of the two phases of $\mathrm{Bi} / \mathrm{GaSb}(110)$, we are of the inclination that the $\mathrm{Bi}$ chains remain intact after the transition from the $(1 \times 1)$ to the $(1 \times 2)$ phase. In Fig. 7 we present one possible overlayer structure of the $(1 \times 2)$ phase. In this model, the geometry of the overlayer consists of a combination of both the ECLS and the EOTS. Neighboring chains couple together with one chain registered above a substrate chain (EOTS), while the other occupies a bridge site above and between two substrate chains (ECLS). This geometry will leave rows of $\mathrm{Sb}$ atoms of the first layer of the substrate, which are between the double chains of the Bi overlayer, with dangling occupied orbitals while satisfying the remainder of the substrate bonds. This overlayer structure will achieve the $(1 \times 2)$ structure, while leaving the chains intact. This geometry has the added advantage that it would introduce the necessary asymmetry to explain the breakdown of the degeneracy of $S^{\mathrm{I}}$ and $S^{\mathrm{II}}$ in the $(1 \times 1)$ phase after the surface transition.

\section{SUMMARY AND CONCLUSIONS}

We have probed the occupied surface bands of the $(1 \times 1)$ and $(1 \times 2)$ phases of $\mathrm{Bi} / \mathrm{GaSb}(110)$ using angle- resolved photoemission spectroscopy with synchrotron radiation. For the $(1 \times 1)$ phase, we have identified four $\mathrm{Bi}$-induced surface bands from lower to higher binding energy $S^{\mathrm{I}}, S^{\mathrm{II}}, S^{\mathrm{III}}$, and $S^{\mathrm{IV}}$, respectively. $S^{\mathrm{I}}$ and $S^{\mathrm{II}}$ were observed to disperse significantly along the $\bar{\Gamma}-\bar{X}$ azimuth, with little dispersion along the $\bar{\Gamma}-\bar{X}^{\prime}$ direction. Consequently, we attribute these bands to intrachain bonds within the Bi overlayer. $S^{\mathrm{III}}$ and $S^{\mathrm{IV}}$ exhibited the opposite behavior with little dispersion in the $\bar{\Gamma}-\bar{X}$ direction, while dispersing significantly along the $\bar{\Gamma}-\bar{X}^{\prime}$ azimuth, which lead us to the conclusion that these bands derive from intrachain bonds which are also involved in the bonding of the overlayer to the substrate.

The integrated intensity of the $\mathrm{Bi} 5 d$ core level was observed to decrease by only $5-15 \%$ during the overlayer transition from the $(1 \times 1)$ to the $(1 \times 2)$ phase, indicating that the $(1 \times 2)$ phase also corresponds to a coverage of 1 monolayer. The surface-state bands $S^{\mathrm{I}}$ and $S^{\mathrm{II}}$ of the $(1 \times 1)$ phase were left intact after the transition to the $(1 \times 2)$ phase. Bands $S^{\mathrm{III}}$ and $S^{\mathrm{IV}}$ of the $(1 \times 1)$ phase appeared to be intact after the surface phase transition, but with significantly reduced bandwidths. From these results we conclude that the $\mathrm{Bi}$ chains of the overlayer remain intact after the transition from the $(1 \times 1)$ to the ( $1 \times 2)$ phase. The degeneracy of $S^{I}$ and $S^{\text {II }}$ at the zone edge $\bar{X}$ lifted after the transition. We surmise from this that a larger asymmetry exists within the overlayer of the $(1 \times 2)$ phase, relative to the $(1 \times 1)$ phase. Based on these results, we propose an overlayer structure of the $(1 \times 2)$ phase which involves the coupling of neighboring Bi chains in a combination of the epitaxial continued layer structure and the epitaxial on-top structure.

\section{ACKNOWLEDGMENTS}

This work was performed at the National Synchrotron Light Source at Brookhaven National Laboratory, which is sponsored by the U.S. Dept. of Energy Division of Materials Sciences and Chemical Sciences. We would like to thank P. Lyman for his assistance at U12B, and L. Kahn for stimulating discussions. A.B.M. would also like to acknowledge partial support from the Natural Science and Engineering Research Council of Canada.
*Permanent address: The Center for Materials Research and Analysis and the Department of Physics, 112 Brace Laboratory, P.O. Box 8801 113, Lincoln, NE 68588-0113.

${ }^{1}$ C. B. Duke, A. Paton, W. K. Ford, A. Kahn, and J. Carelli, Phys. Rev. B 26, 803 (1982).

${ }^{2}$ P. Mårtensson and R. M. Feenstra, Phys. Rev. B 39, 7744 (1989).

${ }^{3}$ A. B. McLean, R. M. Feenstra, A. Taleb-Ibrahimi, and R. Ludeke, Phys. Rev. B 39, 12925 (1989).

${ }^{4}$ T. Guo, R. E. Atkinson, and W. K. Ford, Phys. Rev. B 41, 5138 (1990).

5J. F. McGilp and A. B. McLean, J. Phys. C 21, 807 (1988).
${ }^{6}$ C. M. Bertoni, C. Calandra, F. Manghi, and E. Molinari, Phys. Rev. B 27, 1251 (1983).

${ }^{7}$ P. Skeath, C. Y. Su, W. A. Harrison, I. Lindau, and W. E. Spicer, Phys. Rev. B 27, 6246 (1983).

${ }^{8}$ C. Mailhiot, C. B. Duke, and D. J. Chadi, Phys. Rev. Lett. 53, 2114 (1984); Phys. Rev. B 31, 2213 (1985).

${ }^{9}$ J. P. LaFemina, C. B. Duke, and C. Mailhiot, J. Vac. Sci. Technol. B 8, 888 (1990).

${ }^{10}$ A. Tulke, M. Mattern-Klosson, and H. Luth, Solid State Commun. 59, 303 (1986).

${ }^{11}$ R. M. Feenstra and P. Mårtensson, Phys. Rev. Lett. 61, 447 (1988). 
12P. Mårtensson, G. V. Hansson, M. Lahdeniemi, K. O. Magnusson, S. Wiklund, and J. M. Nicholls, Phys. Rev. B 33, 7399 (1986).

${ }^{13}$ F. Schaffler, R. Ludke, A. Taleb-Ibrahimi, G. Hughes, and D. Rieger, Phys. Rev. B 36, 1328 (1987); J. Vac. Sci. Technol. B 5, 1048 (1987).

${ }^{14}$ A. M. Bowler, J. C. Hermanson, J. P. LaFemina, and C. B. Duke, J. Vac. Sci. Technol. B 8, 1953 (1992).

${ }^{15}$ W. K. Ford, T. Guo, S. L. Lantz, K. Wan, S. L. Chang, C. B. Duke, and D. L. Lesser, J. Vac. Sci. Technol. B 8, 940 (1990).

${ }^{16}$ A. B. McLean, R. Ludeke, M. Prietsch, D. Heskett, D. Tang, and T. M. Wong, Phys. Rev. B 43, 7243 (1991).

${ }^{17}$ A. Samsavar, M. Prietsch, and R. Ludeke (unpublished).

${ }^{18}$ R. D. Meade and D. Vanderbilt, Phys. Rev. Lett. 63, 1404 (1989).

${ }^{19}$ T. Guo, R. E. Atkinson, and W. K. Ford, Rev. Sci. Instrum. 61, 968 (1989).
${ }^{20}$ C. L. Allyn, T. Gustafsson, and E. W. Plummer, Rev. Sci. Instrum. 49, 1197 (1978)

${ }^{21}$ T.-C. Chiang and D. E. Eastman, Phys. Rev. B 22, 2940 (1980).

${ }^{22}$ D. N. McIlroy, D. Heskett, D. M. Swanston, A. B. McLean, R. Ludeke, H. Munekata, and N. J. DiNardo, Phys. Rev. B 47, 3751 (1993).

${ }^{23}$ R. Manzke, H. P. Barnscheidt, C. Janowitz, and M. Skibowski, Phys. Rev. Lett. 58, 610 (1987); R. Manzke and M. Skibowski, Phys. Scr. T31, 87 (1990).

${ }^{24}$ J. Frazedas, M. K. Kelly, and M. Cardona, Phys. Rev. B 43, 2159 (1991).

${ }^{25} \mathrm{G}$. Y. Guo (unpublished).

${ }^{26}$ D. N. McIlroy, D. Heskett, D. M. Swanston, A. B. McLean, R. Ludeke, H. Munekata, and N. J. DiNardo, J. Vac. Sci. Technol. B 11, 1786 (1993). 